\title{
Composite tapered scarf joint repair: analytical model and experimental validation
}

\author{
Xie Zonghong ${ }^{1, a}$, Wang Suian ${ }^{1, b}$ and Li Xiang ${ }^{1, c}$ \\ ${ }^{1}$ School of Astronautics, Northwestern Polytechnical University, Xi'an 710072, China \\ axzhae@nwpu.edu.cn, bnpuwangsuian@gmail.com, ${ }^{\mathrm{c}} 895208876 @ q q . c o m$
}

\begin{abstract}
Keywords: composite laminate; tapered scarf; analytical model; experimental validation
\end{abstract}
Abstract. Analytical models were developed for the analysis of adhesively tapered scarf joint repair subjected to unidirectional tension. Stiffness and strength of the joints were analyzed, and the corresponding analytical equations were presented. Extra plies on repair patch were also considered in the models. A series of specimens with different scarf angles and extra plies were designed to conduct corresponding experiments, as to observe scarf angles and extra pies' influence on the performance of the joints and to validate the results of the models' predictions. The results show that reducing scarf angle and adding extra plies help enhancing the stiffness and strength of the joints within a specified range of scarf angles. The predicted results present a good agreement with the test results. The relative errors of stiffness are less than 5\%, while that of strength are not more than $14 \%$. The proposed analytical models could provide an efficient and accurate tool for parametric study and design of adhesively tapered scarf joints.

\section{Introduction}

The researches and development of composite adhesive bonding repair technique (ABRT) has been continuously growing during the last 50 years owning to the numerous advantages compared with other traditional composite repair techniques, such as riveting and bolting. In other words, ABRT can proffer improved stress distribution in the joint, superior corrosion resistance, ability to join dissimilar materials and other outstanding overall performance[1,2]. Owing to these advantages, ABRT is applied to repair CFRP (Carbon Fiber Reinforced Polymer) panels that undergone damage in the aerospace and automotive industry. One of the ABRT is adhesively scarf joint repair technique, which can maintain the structure's aerodynamic proterties.

In the previous literatures, many works have been devoted on elucidating the mechanical properties about adhesively scarf bonded joints[3,4]. Analysis methods applied to evaluate the scarf joints can be analytical [5] or based on finite element (FE) modeling method[6]. Compared with FE modeling, analytical methods are more efficient and apt to carry on parametric studies. The analytical research for the scarf joints can be traced to Hart-Smith's model[7], which introduced the assumption of elastic-perfectly plastic adhesive, considering the effect of plastic deformation to adhesive film. But he did not treat each composite layer separately, instead of integrating the properties of the composite laminates which were adhesively bonded. Based on Hart-Smith's model, Ahn, et al [8] took into account each individual layer separately for accurate strength prediction of the joints.

The objectives of the present study are twofold. One is to produce an analytical model for tapered scarf joint that can be convenient to obtain the stiffness and strength of a joint subjected with tensile load. The other is to operate corresponding experiments for validating the proposed models. In the present study, Ahn's stepped-scarf models were modified to obtain the analytical model for tapered scarf joint. A modified criterion was also applied to analyze the strength of the joint by considering the peak stresses across the adhesive bond line.

\section{Analytical model}

Usually, the scarf joint repair technique requires the elimination of a certain amount of the undamaged structure surrounding the damaged area so that the repair material can be inserted into the 
laminate damaged. Either wet resin lay-up or prepreg materials can be used as the repair patch. There are two different preparation styles for scarf repair, including stepped scarf repair and tapered scarf repair. The latter is what this study focused on, which is shown in Fig 1. Both the BL and the RP are assumed to behave in linearly elastic manner, while the adhesive layer exhibited elastic-perfectly plastic behavior [8].

The tangent of scarf angle $\beta$ was defined as taper ratio between the thickness of BL $H$ and lap length $d_{s}$, while $h_{s}$ is defined as step height. As seen, the smaller $h_{s}$, the denser the total steps are and the stepped scarf joint get closer to tapered scarf joint. The correlation between $h_{s}$ and taper-scarf structure is to be discussed in Section 1.3.

Building an analytical model for tapered scarf joint is extremely difficult since the centerline of the adhesive is not parallel with the midline of the BL or RP. One of the methods is adapting the stepped scarf joint model for tapered scarf joint model by choosing an appropriate $h_{s}$.

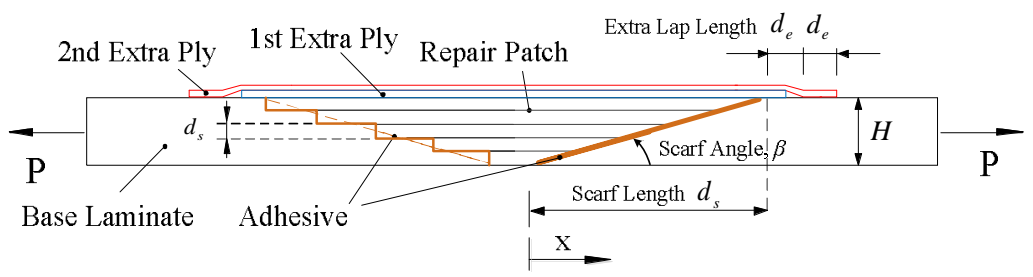

Fig 1 Tapered scarf joint repair

Shear strain expressions for stepped scarf joint

Since the geometry is symmetric, the scarf joint can be simplified to half of the structure shown in Fig 2. All steps are numbered from 1 to $K$. The adhesive length of $i-t h$ step is defined as ${ }^{i} d_{k}$. The joint is subjected to a tensile load $P$. According to force balances for a $d x$ long section and deformation correlation, the expressions for the shear strain in the adhesive can be obtained.

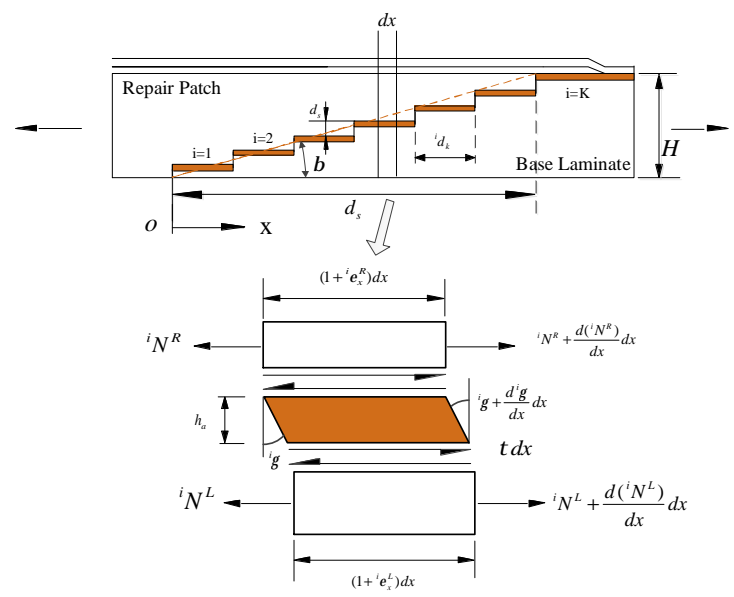

Fig 2 Loads analysis on dx section of the laminate repaired by scarf technique

In the elastic region, the shear stress expression for $i-t h$ segment is

$$
{ }^{i} \gamma_{e}(x)={ }^{i} \gamma={ }^{i} A \sinh \left({ }^{i} \lambda x\right)+{ }^{i} B \cosh \left({ }^{i} \lambda x\right)
$$

Where $\lambda=\sqrt{\frac{G}{h_{a}}\left({ }^{i} a_{11}^{L}+{ }^{i} a_{11}^{R}\right)}$.

In plastic region, ${ }^{i} \tau$ is constant and equal with $G \gamma_{e f}$, so yields another general solution. 


$$
{ }^{i} \gamma_{p}(x)={ }^{i} \gamma=\frac{\left({ }^{i} \lambda\right)^{2} \gamma_{e f}}{2} x^{2}+{ }^{i} r x+{ }^{i} s
$$

${ }^{i} a_{11}$ is the 11 component of the $\mathrm{BL}$ or RP compliance matrix in the $i-t h$ segment; the right superscripts $L$ and $R$ refer to BL and RP; the left superscript $i$ refers to $i$-th segment; $G$ is the shear modulus of the adhesive; $h_{a}$ is the thickness of the adhesive ${ }^{i} A,{ }^{i} B,{ }^{i} r$ and ${ }^{i} s$ in the Eq. 1 and 2 are unknown constants which can be determined from the boundary and continuity conditions, which are presented in literature[8].

Analyses of the laminate and the joint's stiffness

The shear strain expression can be used to evaluate the failure level of the adhesive. However, to assess the strength of the scarf joint, mechanical analyses for BL and RP should be made. When the shear strain expression ${ }^{i} \gamma(x)(i=1$ to $K)$ is given, it easy to get the in-plane loads in BL $\left({ }^{i} N^{L}(x)\right)$ and $\mathrm{RP}\left({ }^{i} N^{R}(x)\right)$, which are the functions of axial position $x$

$$
\begin{aligned}
& { }^{i} N^{L}(x)=0+\int_{0}^{x} \tau(\zeta) d \zeta \\
& { }^{i} N^{R}(x)=P-\int_{0}^{x} \tau(\zeta) d \zeta
\end{aligned}
$$

Through the corresponding compliance matrix and transformer matrix, the on-axis in-plane strains of each ply in the BL or in the RP in $i-t h$ step can be obtained. Finally, an appropriate failure criterion is applied to assess the failure degree of the BL and RP.

Fig. 3 demonstrates the deformation of the joint. The total deformation consists of longitudinal deformation of BL and RP in undamaged region, longitudinal deformation of BL or RP in scarf region and shear deformation of adhesive. So the total deformation for a scarf joint long as $2 L$ can be expressed as

$$
\Delta=2 \times\left(\int_{0}^{d_{R}}{ }^{0} \varepsilon_{11}^{R}(x) d x+\sum_{i=1}^{K} \int_{0}^{i d_{k}}{ }^{2} \varepsilon_{11}^{L}(x) d x+\int_{0}^{d_{L}}{ }^{0} \varepsilon_{11}^{L}(x) d x+{ }^{1} \gamma h_{a}\right)
$$

The stiffness of the scarf joint is obtained

$$
E_{\text {stif }}=\frac{\Delta \sigma}{\Delta \varepsilon}=\frac{P \cdot 2 L}{A \cdot \Delta}
$$

Where $A$ is the cross-sectional area of BL.

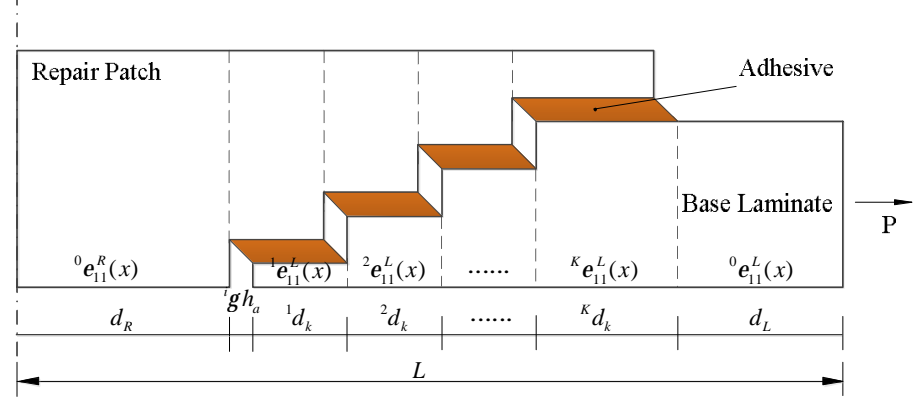

Fig 3 Illustration of the deformation of scarf joint

Equivalent model for tapered scarf joint

As mentioned above, with increasing the numbers of total steps of the stepped scarf joint or reducing the step length $h_{s}$, the stepped scarf structure is gradually approaching the tapered scarf joint in appearance. The shear strain/stress field is the key property affects the reliability of the adhesive, thus it 
is reasonable to observe the change of the shear strain field to evaluate the practicability of this equivalent method.

The change of the shear strain and the peak shear strain of the adhesive are demonstrated in Fig. 5, with $h_{s}$ varying from $0.125 \mathrm{~mm}$ (ply thickness of BL or RP) to $0.018 \mathrm{~mm}$. As seen, with the decrease of $h_{s}$, the shear strain field tend to converge into a unique field, and the maximum shear strain approach a specific value rapidly. Theoretically, the smaller $h_{s}$, the more accurate the equivalent model. However, exorbitant step numbers may make the numerical error soars, resulting in erroneous results. Actually, a $h_{s}$ less than $0.03 \mathrm{~mm}$ is enough, as is shown is Fig. 4, which not only maintains the accuracy of tapered scarf analytical model, but also guarantee calculating efficiency and numerical errors. Here, the stepped scarf model with $h_{s} \leq 0.03 \mathrm{~mm}$ is used as the tapered scarf model.

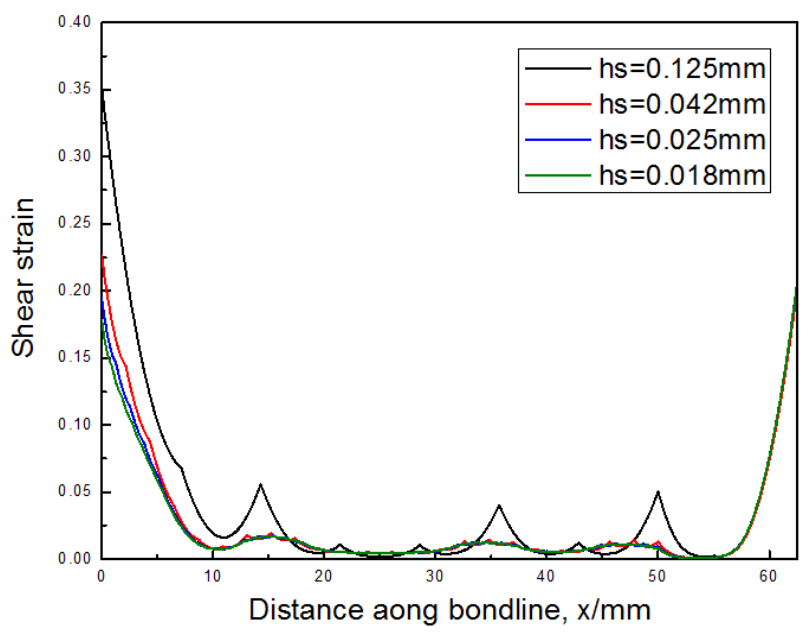

(a) Shear strain field

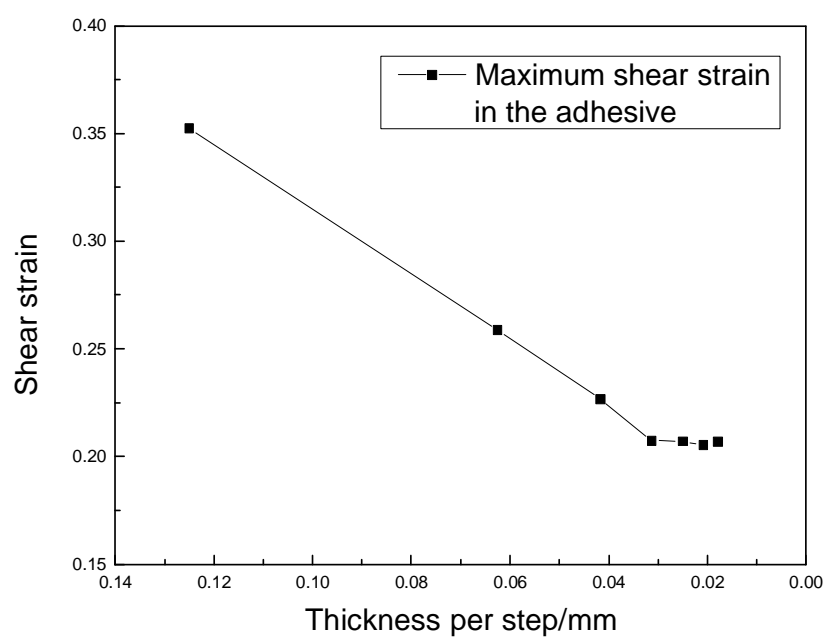

(b) Maximum shear strain

Fig 4 The change of shear strain field/maximum shear strain with

\section{Experiment}

In this study, materials for the specimens were all produced by Cytec. The adhesives in the specimens were made of METLBOND 1515-4M, a stiff adhesive. T300/CYCOM 970, a fiber reinforced weave, were used to manufacture $\mathrm{BL}$ or $\mathrm{RP}$, which were cured by auto-clave for $\mathrm{BL}$ or cured by hot-bonder for RP. The mechanical properties of the specimens' BL, RP and adhesive were tested in accordance with the ASTM standards given in Table 1.

Eight configurations were employed in the experiments, including specimens with one extra ply and specimens with two extra plies and with design scarf angles ranging from $2^{\circ}$ to $5^{\circ}$ (actual scarf angles are listed in Table 4$)$. The BL and RP are made with the same stacking sequence $\left([0 / 45]_{2 S}\right)$, whilst the ply thickness is $0.210 \mathrm{~mm}$. And the measured thickness of adhesive film is approximately $0.125 \mathrm{~mm}$. Other geometrical parameters were shown in Fig. 5.

Table 1 Mechanical properties of the laminates and the adhesive film

\begin{tabular}{llll}
\hline Mechanical properties & T300/CYCOM 970(Autoclave) & T300/CYCOM 970(Hot-bonder) & Metlbond 1515-4M \\
\hline$E_{1}=E_{2}(\mathrm{GPa} a)$ & 66.5 & 59.1 & 3.57 \\
$G_{12}(\mathrm{GPa})$ & 4.7 & 3.9 & 0.37 \\
$\mathrm{v}_{12}$ & 0.07 & 0.05 & \\
$X_{t}=Y_{t}(\mathrm{MPa} a)$ & 611.1 & 510.6 & \\
$S(M P a)$ & 121.3 & 113 & 13.8 \\
$\tau_{p}(\mathrm{MPa})$ & & & 0.30 \\
$\gamma_{p f}$ & & & \\
\hline
\end{tabular}




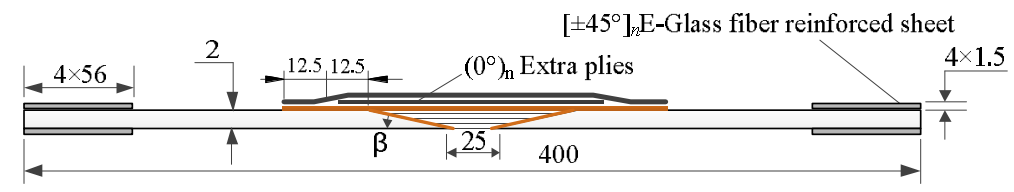

Fig 5 Geometric parameters of adhesively taper-scarf joint

\section{Results and discussions}

The repair joints were tested in tension using a 'HuaLong' testing machine at a constant crosshead rate of $1 \mathrm{~mm} / \mathrm{min}$. The results were listed in Table 2 .

The measured data of stiffness is stable and reliable, of which the coefficients of variation (CV) are pretty small (mostly below 5\%). There are no significant changes observed for the joints' stiffness, of which the stiffness recovery ratio (joint's stiffness/BL's stiffness) ranges from $64.8 \%$ to $76.2 \%$.

The strength recovery ratio (joint's strength/BL's strength) of the specimens ranges from $34.9 \%$ to $67.6 \%$. The strength of tapered-scarf repair joints is negatively related to scarf angle in the range from 1.5 degree from 5 degree. Adding the number of extra ply has an obvious strength promotion to the joints. Under the same scarf angle, the joints with two extra plies obtain an average strength promotion of $44.7 \mathrm{MPa}(7.3 \%)$ than the joints with one extra ply.

Table 2 Specimen number and tensile test results of tapered scarf joint repair

\begin{tabular}{|c|c|c|c|c|c|c|c|c|}
\hline \multirow{2}{*}{ No } & \multirow{2}{*}{$\begin{array}{l}\text { Number of } \\
\text { specimens }\end{array}$} & \multicolumn{2}{|c|}{ Scarf angle } & \multirow{2}{*}{$\begin{array}{l}\text { Nums of } \\
\text { extra plies }\end{array}$} & \multicolumn{2}{|c|}{ Stiffness(GPa) } & \multicolumn{2}{|c|}{ Strength(MPa) } \\
\hline & & Design & Actual & & Average & $\mathrm{CV}(\%)$ & Average & $\mathrm{CV}(\%)$ \\
\hline B1 & 3 & \multirow{2}{*}{2} & \multirow{2}{*}{1.75} & 1 & 45.7 & 0.9 & 406 & 4.3 \\
\hline B2 & 3 & & & 2 & 50.7 & 1.0 & 413 & 2.9 \\
\hline $\mathrm{C} 1$ & 3 & \multirow{2}{*}{3} & \multirow{2}{*}{2.63} & 1 & 43.3 & 1.1 & 342 & 5.6 \\
\hline $\mathrm{C} 2$ & 3 & & & 2 & 46.0 & 2.6 & 353 & 7.5 \\
\hline D1 & 3 & \multirow{2}{*}{4} & \multirow{2}{*}{3.50} & 1 & 44.6 & 2.6 & 263 & 7.5 \\
\hline D2 & 3 & & & 2 & 46.0 & 1.2 & 335 & 11.1 \\
\hline E1 & 3 & \multirow{2}{*}{5} & \multirow{2}{*}{4.38} & 1 & 43.1 & 0.5 & 213 & 8.1 \\
\hline $\mathrm{E} 2$ & 3 & & & 2 & 45.6 & 0.6 & 302 & 16.4 \\
\hline
\end{tabular}

By the analytical model of tapered scarf joint above, the corresponding theoretical results can be calculated. Maximum Strain Failure Criterion is applied to evaluate the failure degree of the BL and the RP, while Damage Zone Theory [9] is adopted to assess the adhesive. The predictions will be compared with the experimentally measured stiffness and failure loads.

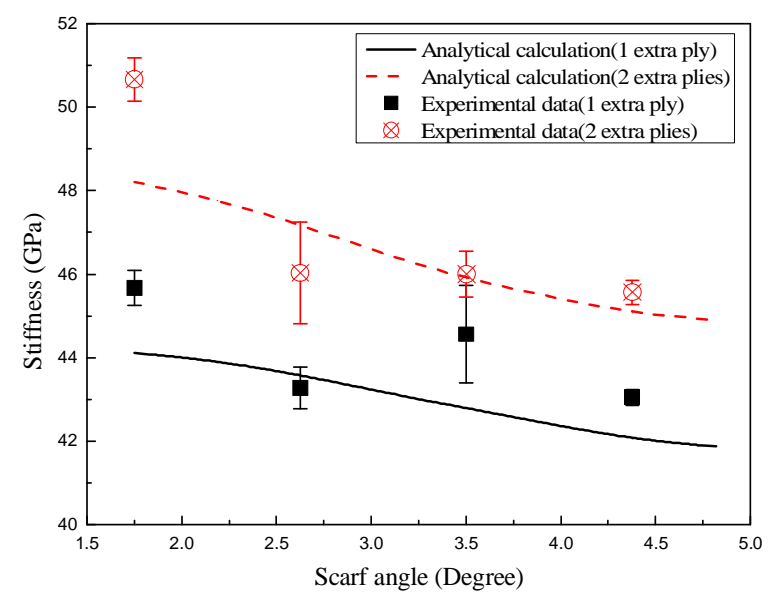

(a) Stiffness

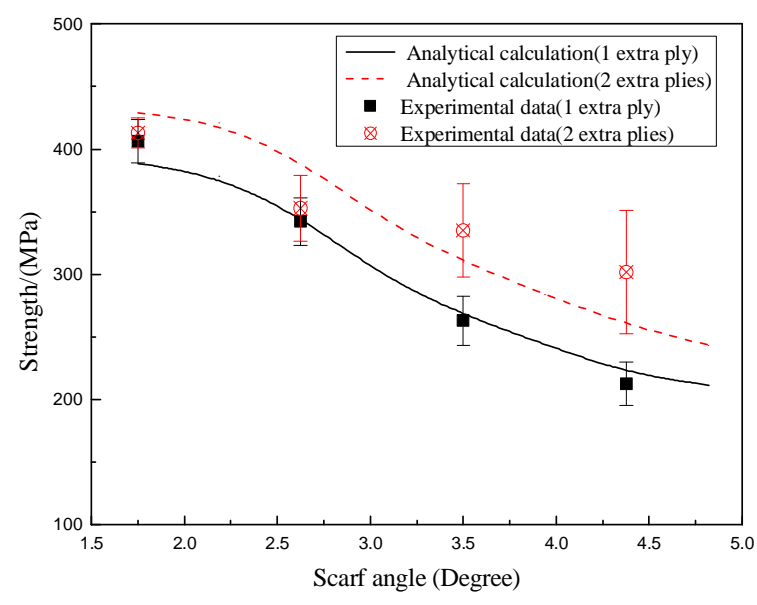

(b) Strength

Fig 6 Comparison of the joints' stiffness and strength with various scarf angles 
Fig. 6(a) shows the stiffness versus scarf angle curve, while the test points are scattered for comparison. A small decrease was observed as the scarf angle increases for both analytical and test results. The stiffness predicted and measured are in close agreement, for the relative errors are less than $4.8 \%$.

The strength versus scarf angle curves are plotted in Fig. 6(b). When scarf angle increases, there is significant strength decrease for joints with one extra ply of two extra plies. It is evident that the experimental results are perfectly in accordance with the predicted curve for the joints with one extra ply. The maximum relative error is merely $4.5 \%$, which is pretty small. By contrast, the results for the joints with two extra plies don't agree so well (maximum relative error 14.0\%). The joints with two extra plies can achieve an increased strength of $41.4 \mathrm{MPa}$ than the joints with one extra ply on average. The value is quite consistent with the experimental results (44.7MPa).

\section{Conclusions}

In this study, the mechanical behaviors of eight different configurations of tapered scarf joints subjected to tensile load were investigated by analytically and experimentally.

Analytical model for tapered scarf joint repair is presented, carrying out parametric analyses for stiffness and strength of the joints, and discussing the practicability of the equivalent method.

It is found from tests that for joints having the same extra ply, stiffness and strength are negatively related to scarf angle in the range from 1.5 degree from 5 degree. But the scarf angle's effect on joints' strength is more significant for strength than that on stiffness. Joints having the same scarf angle with two extra plies obtain an average strength promotion of $44.7 \mathrm{MPa}(7.3 \%)$ than that with one extra ply.

Comparisons of test results and predicted results show a good agreement. The relative errors of stiffness are all less than 5\%, while that of strength are not beyond $14.0 \%$. Accordingly, the proposed analytical model for tapered scarf joint repair is efficient and practical, providing a fast assistance tool for parametric analysis and design of composite laminate adhesively bonded repair.

\section{Acknowledgements}

The work is supported by National Natural Science Foundation of China (Grant No. U1233202)

\section{References}

[1]. Banea M D, da Silva L F M. Adhesively bonded joints in composite materials: an overview[J]. Part L: Journal of Materials Design and Applications, 2009, 223(1): 1-18.

[2]. Katnam K B, Da Silva L F M, et al. Bonded repair of composite aircraft structures: A review of scientific challenges and opportunities[J]. Progress in Aerospace Sciences, 2013, 61: 26-42.

[3]. Soutis C, Hu F Z. Failure analysis of scarf-patch-repaired carbon fiber/epoxy laminates under compression[J]. AIAA journal, 2000, 38(4): 737-740.

[4]. Bendemra H, Compston P, Crothers P J. Optimisation study of tapered scarf and stepped-lap joints in composite repair patches[J]. Composite Structures, 2015, 130: 1-8.

[5]. Erdogan F, Ratwani M. Stress distribution in bonded joints[J]. Journal of Composite Materials, 1971, 5(3): 378-393.

[6]. Harman A B, Wang C H. Improved design methods for scarf repairs to highly strained composite aircraft structure[J]. Composite structures, 2006, 75(1): 132-144.

[7]. Hart-Smith L J. Adhesive-bonded single-lap joints[M]. VA: Langley Research Center, 1973.

[8]. Ahn S H, Springer G S. Repair of Composite Laminates-II: Models[J]. Journal of Composite Materials, 1998, 32(11):1076-1114. 
[9]. Ban C S, Lee Y H, Choi J H, et al. Strength prediction of adhesive joints using the modified damage zone theory[J]. Composite Structures, 2008, 86(1-3):96-100. 